\title{
Combined Teletraffic/Transmission Performance of Optical Cross-connected Networks using Hybrid-Store-and-Forward
}

\author{
G. A. Castañón, O. K. Tonguz \\ Photonics Research Laboratory \\ Department of Electrical and Computer Engineering \\ State University of New York at Buffalo, Buffalo, NY 14260, USA
}

\author{
A. Bononi \\ Dipartimento di Ingegneria dell'Informazione \\ Università di Parma, I-43100 Parma ITALY
}

\begin{abstract}
Transparent multihop optical networks suffer from the accumulation, from node to node, of intraband crosstalk and amplifier spontaneous emission noise, which may severely degrade the quality of received signals. This paper presents a hybrid semitransparent store-and-forward (H-S\&F) node architecture which minimizes the number of hops and, therefore, significantly improves the quality of signals. Cells are electronically stored just in the case of conflict to avoid deflection, otherwise cells traverse the node without opto-electronic conversion. We present the teletraffic and transmission performance of regular two-connected networks in uniform traffic. Manhattan Street (MS) Network and ShuffleNet (SN) are compared both analytically and by simulation. HS\&F performs well, in terms of throughput, transit delay and BER. It is also shown that by combining deflection routing with the storeand-forward scheme the network can accommodate two different bit-rates. This suggests that the proposed hybrid scheme may have good potential for future multimedia networks.
\end{abstract}

\section{INTRODUCTION}

The major advantage of cross-connected optical networks is that they achieve higher throughput than linear topologies like buses and rings [1], [2]. If buffers are not available, the cells can be temporarily deflected to an undesired link. Thus, deflection routing allows the use of fiber links as optical buffers [1]-[3] while bit-rate non-regenerative transparency is maintained. Such an advantage in traffic management causes a major weakness in transmission [4]. It has been shown [5] that the quality of signals degrades with traffic load due to accumulation of weak noises such as the amplifier spontaneous emission (ASE) noise and coherent crosstalk in high-speed transparent optical networks. Therefore, node architectures that limit the propagation delay to a minimum average number of hops and keep a certain bit-rate transparency are ideal and, therefore, desirable for cross-connected networks. Here we present and analyze one of such semi-transparent node architectures. Such an architecture avoids deflections by providing internal electronic buffers. Cells are stored just in the case of conflict, to avoid deflection, otherwise the cells will transparently traverse the node (transparent cut-through [6] routing). Buffered cells only are regenerated by the intermediate nodes.

We analyze the performance of this node architecture under two access schemes: a) buffered cells have access priority; b) locally generated cells have access priority. These two access schemes have a largely different impact on the queue size and, therefore, on the queueing delay. Also, we will show that this node architecture can sustain a two bit-rate communication if a combination of hybrid store-and-forward and deflection routing is used.

This paper analyzes the steady-state behavior of two connected mesh networks under a hybrid-store-and-forward (H-S\&F) scheme. The analytical teletraffic model in [3] is reviewed and extended to hybrid-S\&F. Also, we present results of the transmission performance based on the traffic randomness of multi-hop cell-switched multiwavelength networks at the optimal transmission power. We present the limit of operation based on a uniform traffic scenario. The main impairments considered in the transmission analysis are intra-band crosstalk and ASE noise.

\section{NODE STRUCTURE}

The node is composed of a stack of independently-operated submodules, one per each wavelength. The wavelengths from the input fibers are spatially demultiplexed and sent to the appropriate submodule for add/drop operations and switching. Cells from the submodules are finally re-multiplexed onto the output fibers. Fig. 1a shows a hybrid structure that employs two electronic buffers with sufficient capacity. The header recognition block taps power off to electronically read the cell header and make routing/control decisions. Each submodule is equipped with one transmitter (TX) and two receivers (RX). Cells transparently flow through the node and are stored only in case of conflict. This avoids both deflection and repetitive optical/electronic conversion (as in conventional S\&F). Stored cells are transmitted using a first-in-first-out (FIFO) scheme. Buffered cells are regenerated by the intermediate nodes. The logical structure of the node is shown in Fig. 1b. The logical flow of node operations is absorption, buffering/injection, and routing.

When a cell is routed through a node, one of the two outputs is chosen according to a shortest path algorithm [7]. Based on the position of the intermediate node and the cell's destination node, one or both outputs may be suitable for minimizing the number of hops a cell has to traverse for reaching destination. A cell that can take both outputs is called a don't care cell, while a cell that has only one preferred output is called a care cell. Basically, slots can be empty (E), can carry a cell for the node (FN), or a cell that cares to exit at output 1 (C1) or output 2 (C2), or a don't care (DC) cell.

Now let's briefly define some teletraffic parameters. Define $u$ as the input slot utilization, i.e., the probability that an input slot carries a cell. Define $P_{d c}$ as the probability that an incoming cell is 


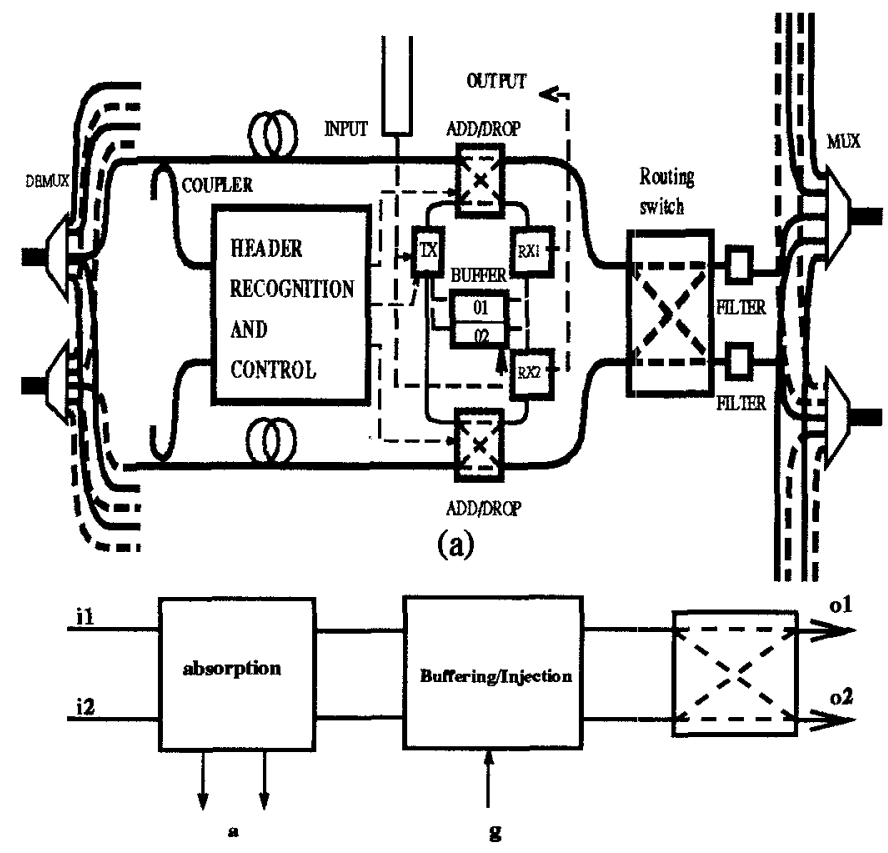

(b)

Figure 1: a) Node and submodule block diagram, submodule stores cells just in the case of conflict, b) Logical submodule structure.

DC. Let $a$ be the probability that an input cell is destined to the node. The probability of cell absorption $a$ is related to the average propagation delay $H$ (in number of hops) as: $a=1 / H$ [3]. We will assume that, at every time-slot $t$, the input arrivals $i_{1}(t)$, $i_{2}(t)$ (of one wavelength) are independent random variables with the same probability distribution $\mathbf{f}_{\mathbf{i}}=\left\{\operatorname{Pr}\left[i_{j}=s\right], s \in\{E, F N\right.$, $D C, C 2, C 1\}\}, j=1,2$. From the above definitions one gets after the absorption block: $\mathbf{f}_{\mathbf{i}}=\left\{f_{i}(E), f_{i}(F N), f_{i}(D C), f_{i}(C)\right\}$ $=\left\{1-u(1-a), u a, u(1-a) P_{d c}, u(1-a)\left(1-P_{d c}\right)\right\}$ [3]. Also define $A 0, A 1$, and $A 2$ as the probabilities of having respectively 0,1 or 2 cells in one wavelength (or submodule) after the absorption block, whose expressions are

$$
\begin{aligned}
& A 0=(1-u(1-a))^{2} \\
& A 1=2 u(1-u)(1-a)+2 u^{2} a(1-a) \\
& A 2=u^{2}(1-a)^{2} .
\end{aligned}
$$

To keep the analysis simple, we assume that each TX has no local input queue. New cells per wavelength are generated in each time slot with probability $g$, the generation probability. If a new cell is generated but can not be injected into the network, local blocking occurs and the local cell is discarded.

\section{ACCESS SCHEMES}

We will assume that successive slot-by-slot arrivals are independent. This assumption is partially violated when use is made of buffers and the transmission priority is given to the locally generated cells by the submodule since, at high loads, the buffers tend to correlate successive arrivals [8], [9]. This successive cell correlation causes one of the buffers of a generic submodule to be filled at a faster rate than the other, thus generating imbalance in the queues and extra queue size and queueing delay. This successive cell correlation depends on the traffic load and affects ShuffleNet [8] at high traffic loads ( $g>0.7$ ) while Manhattan Street is little affected as we will show. However, when access priority is given to the routing buffers, the degree of correlation of successive arrivals is small for both SN and MS. Therefore, the model presented here, which assumes that the arrivals on different links are independent, is fairly accurate. We present simulation results to validate the accuracy of the model according to the method discussed in [10], which we extend to hybrid-S\&F.

\subsection{Hybrid-S\&F: Buffered cells have access priority}

In this section we will derive the average queue size and the queueing delay when cells stored in the electronic routing buffers have access priority over cells generated by the submodule. The two queues (01 and 02) of Fig. 1a can be modeled as independent birthdeath Markov chains, each with birth rate $\beta$ and death rate $\mu$. The average queue size (in number of cells) of one buffer is given by [11] $Q_{b}=\frac{\beta}{\mu-\beta}$, where

$$
\begin{aligned}
\beta=\frac{1}{2} \frac{\left[A 2+g q^{2} A 1\right]\left(1-P_{d c}\right)^{2}}{2} \\
\mu= \\
+\frac{1}{2} \frac{A 2\left(1-P_{d c}\right)^{2}}{2} .
\end{aligned}
$$

The symbols have the following meaning. $\beta$ is the probability to store a cell in one buffer. A cell is stored just in the case that two care cells desire the same output link (to avoid deflection). This event will occur when there are two $(A 2)$ incoming care $\left(1-P_{d c}\right)$ cells at the links of the submodule, with the same output preference $(1 / 2)$, or the submodule generates a new care cell whenever the buffers are empty $\left(q^{2}\right)$ and there is one $(A 1)$ incoming care cell with the same output preference $(1 / 2)$ as the locally generated cell. If this last event occurs, the locally generated cell is routed electronically to the buffers (see Fig. 1a) instead of storing the cell that is in transit. The first (1/2) factor in (2) is the probability to store a cell in buffer 01 or $02 . \mu$ is the probability of buffer transmission. This will occur if the second buffer is empty $(q)$, or the second buffer is not empty and there is $1 / 2$ probability of buffer selection and there are two free slots $(A 0)$ or one $(A 1)$ don't care cell is present $\left(P_{d c}\right)$. The second term refers to the probability that one incoming care cell is present targeting the same output as the stored cell with probability $1 / 2$. The last term refers to the probability that a cell is stored in the second buffer. Also in this case buffered cells have access priority over locally generated cells. Due to the symmetry of the networks, and considering that the traffic is uniform, we assume the same $\beta, \mu$, and $q$ for both queues of each submodule.

The probability that one of the buffers is empty $(q)$, is given by [11] $q=\frac{\mu-\beta}{\mu}$, and the average queueing delay produced by one buffer is [11] $D_{b}=\frac{1}{\mu-\beta}$.

Applying Little's theorem to one optical channel of the network including the buffers, the balance equation is $\lambda H=2 \aleph u$ where $\lambda$ is the network's per channel throughput, i.e. the average number of cells inserted/absorbed per slot per channel in the network at equilibrium. $\aleph$ is the number of submodules per channel.

The average number of newly transmitted (injected) cells per submodule is obtained as the probability of having a new cell ready for 
transmission $(g)$ times the probability that both buffers are empty $\left(q^{2}\right)$ times the probability that at least one of the two slots is free:

$$
\frac{\lambda}{\aleph}=g q^{2}\left(1-u^{2}(1-a)^{2}\right) \text {. }
$$

Then, it is easily shown that a closed form expression for $\mathrm{u}$ is

$$
u=\frac{\sqrt{a^{2}+\left(g q^{2}\right)^{2}(1-a)^{2}}-a}{g q^{2}(1-a)^{2}},
$$

and the total average transit delay, that is the sum of the propagation delay $H$ and the possible internal queueing delay normalized by the hop propagation delay, is

$$
d=H\left(1+\frac{D_{b} \rho}{W}\right)
$$

where $W=\frac{l}{c / n} \frac{R}{M} \cong 11.75 R[G b / s] l[\mathrm{~km}]$ is the ratio of link length to the spatial length of one slot [3], where $l$ is the link length, $c / n$ is the light speed in optical fibers of refraction index $n=1.5$, $R$ is the bit rate and $M$ the cell size (424 bits). $H D_{b} \rho$ is the possible queueing delay, where $\rho=\frac{1}{4} u(1-a)\left(1-P_{d c}\right)^{2}$ is the probability of buffering a cell.

The values of the average number of hops $(H)$ and probability of don't care $\left(P_{d c}\right)$ can be obtained considering a probability of cell deflection $p=0$ (for this case) and that the random walk of a test cell toward destination is modeled as an absorbing Markov chain whose states are defined by the network nodes, the only absorbing state being the destination node as in [3], [12].

\subsection{Hybrid-S\&F: locally generated cells have access priority}

In this section we will derive the average queue size and the average queueing delay for the case of Hybrid-S\&F when cells generated by the submodule have access priority over cells stored in the buffers. In this case $\beta=\frac{1}{2} \frac{[A 2+g A 1]\left(1-P_{d c}\right)^{2}}{2}$ and $\mu=(q+$ $\left.\frac{1-q}{2}\right)(1-g)\left(A 0+A 1 P_{d c}\right)+\frac{(1-g) A 1\left(1-P_{d c}\right)}{2}+\beta$, where $\beta$ and $\mu$ can be obtained by reasoning as in equations (2) and (3), except that in this case $\beta$ is not conditioned on the buffer being empty, and $\mu$ is conditioned on the probability that no new cells from the submodule are present for transmission $(1-g)$. In this case the link utilization $u$ is given by $u=\frac{\sqrt{a^{2}+g^{2}(1-a)^{2}}-a}{g(1-a)^{2}}$. Also, to compute the transit delay $d$, eq. (6) can be used.

Fig. 2 shows throughput versus probability of cell generation $g$ for MS and SN topologies. When access priority is given to the locally generated cells (N-P) MS network gives a high throughput. For the case of SN network the throughput given by the simulation starts to decay at $g=0.75$ due to the fact that cell correlation produces a higher number of conflicts at the nodes, this means that more cells are stored/extracted in/from the buffers, therefore less new cells are injected and throughput decays. This fact indicates that cell correlation is much more severe in SN64 than in MS64. When access priority is given to the routing buffers (B-P) the throughput is $\lambda=21$ for MS and $\lambda=22.1$ for SN at $g=1$. Also, from this figure we can observe that the throughput given by B-P is slightly better at high loads to the one given by one-buffer and better than hot-potato deflection routing. All simulation statistics were collected for 30,000 clock cycles, after discarding 10,000 initial cycles to allow for transients to die out.

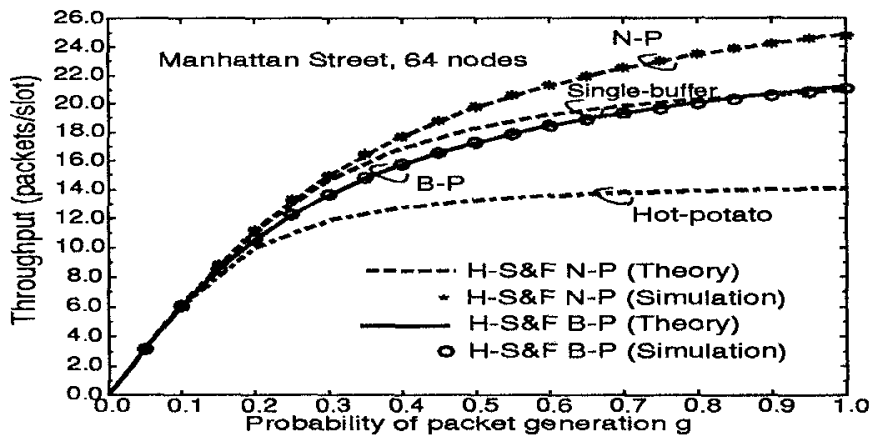

(a)

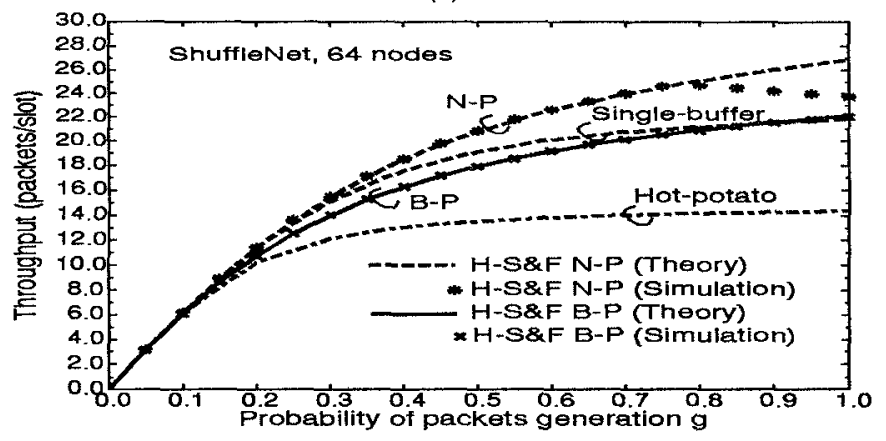

(b)

Figure 2: Throughput number of hopsvs. $g$ for a) Manhattan Street and b) ShuffleNet topologies.

Fig. 3 shows the average number of cells (queue size $Q_{b}$ ) stored in one of the two internal buffers of Fig. 1a and the average queueing delay $D_{b}$ in number of cells for MS and SN networks with 64 nodes. When access priority is given to the routing buffers (BP) the average queue size is smaller than 1 cell and the average queueing delay is lower than 4 cells for both topologies. When access priority is given to locally generated cells (N-P) the queue size and queueing delay are reasonable for MS at loads lower than $g=0.95$. Observe that theory and simulation results show a good agreement for MS topology, while results for SN (N-P) present a mismatch between the theory and simulation at loads higher than $g=0.7$. The reason for this discrepancy is that buffers tend to correlate the cells and buffer imbalance is produced generating extra queue size and queueing delay.

It is important to mention that the total cell delay is the sum of its transit delay $d$ (propagation delay in the network plus possible queueing delay of the internal buffers), and the input queueing delay [see Appendix A]. Fig. 4 shows the transit delay $d$ (in hops) and the input queueing delay (in cells or slots) against the throughput per submodule for H-S\&F both cases B-P, N-P, and also for hot-potato and one-buffer deflection routing [3]. When access priority is given to the locally generated cells (N-P) the average transit delay of MS increases at high loads $(g>0.95)$ due to internal queueing delays. When access priority is given to the routing buffers (B-P) the average transit delay is about 5 and 4.7 hops for MS and SN respectively. Observe that the internal queueing delay is minimized because the internal buffers have access priority. The $\mathrm{H}-\mathrm{S} \& \mathrm{~F}$ average internal queueing delay of a generic cell during its travel to destination is given by $H D_{b} \rho$ cells (or slots). If one considers the worst case, in which a cell is successively stored at each node (then $\rho=1$ ), the total internal buffer delay is about 20 cells 


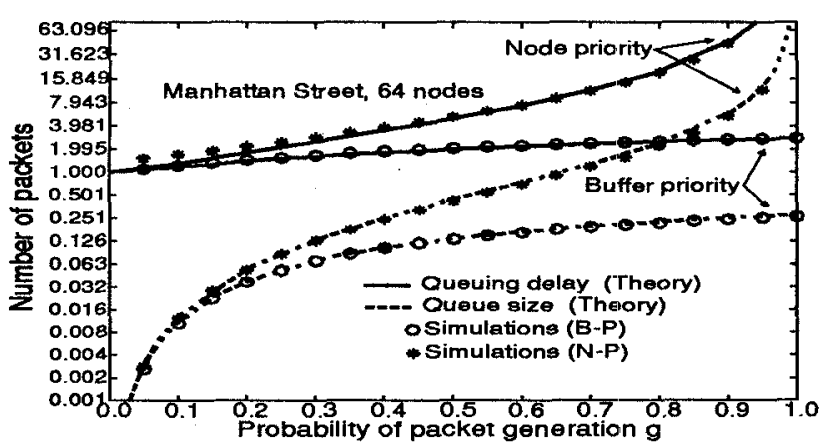

(a)

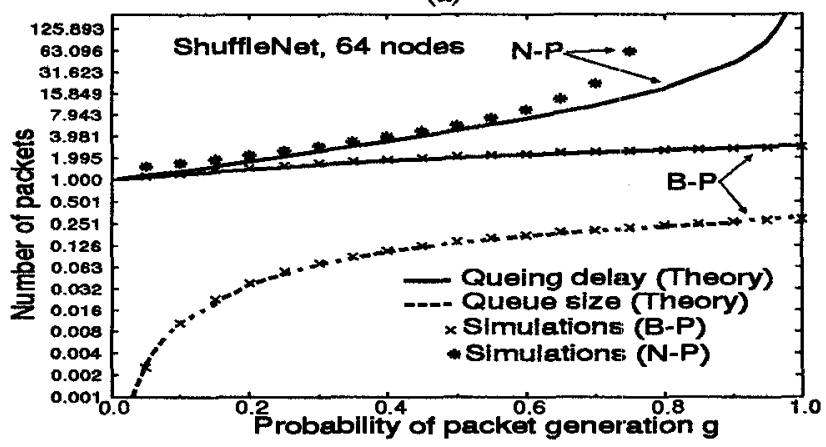

(b)

Figure 3: Queueing delay and Queue size in number of cells vs. $g$ for a) Manhattan Street and b) ShuffleNet topologies with 64 nodes.

for MS and 19 cells for $\mathrm{SN}$ at $\mathrm{g}=1$. This shows that $\mathrm{B}-\mathrm{P}$ access scheme reduces the cell inter-arrival time jitter (delay jitter), however this comes at expense of reducing the throughput with respect to N-P. Also, observe that the input queue reach saturation at the maximum throughput per submodule for all cases as is intuitively expected.

\subsection{Hybrid S\&F-deflection routing: Transmission at two different} bit rates

Now suppose that $N_{R 2}$ submodules on one optical channel want to upgrade their bit-rate to bit-rate-2 $(R 2)$ and $N_{R 1}$ submodules remain at bit-rate-1 $(R 1)(R 2>R 1)$. Assume, for analytical convenience, that $R 2=m R 1$, where $m$ is an integer. If a submodule that transmits at $\mathbf{R} 2$ wants to send a cell to a submodule that receives at bit-rate $R 1$, it has to repeat ones or zeros $m$ times each. Then the actual bit rate of the connection is R1. Conversely, if the transmission is from slow submodules $R 1$ to fast submodules $R 2$, the fast receiver, which integrates over its bit time $\frac{1}{R^{2}}$, has to be able to collect $m$ samples before making a decision. This is called oversampling, and has the effect of decreasing the error rate, even though the receiver has more noise because of the larger receiver bandwidth. For example, if $m$ is even, the new bit error rate is

$$
\begin{aligned}
B E R_{m-e v e n} & =\left(\begin{array}{c}
m \\
b
\end{array}\right)_{b=\frac{m}{2}} \frac{S E R^{b}(1-S E R)^{m-b}}{2} \\
& +\sum_{b=\frac{m}{2}+1}^{m}\left(\begin{array}{c}
m \\
b
\end{array}\right) S E R^{b}(1-S E R)^{m-b}
\end{aligned}
$$

and if $m$ is odd then

$$
B E R_{m-o d d}=\sum_{b=\left\lceil\frac{m}{2}\right\rceil}^{m}\left(\begin{array}{c}
m \\
b
\end{array}\right) S E R^{b}(1-S E R)^{m-b}
$$

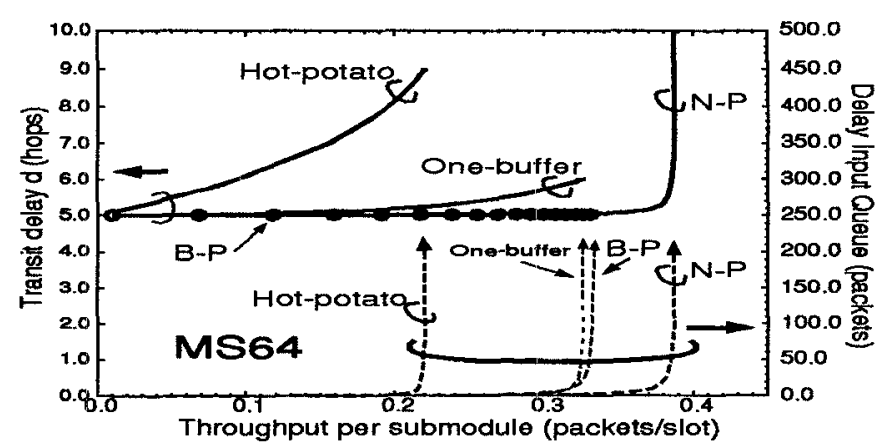

(a)

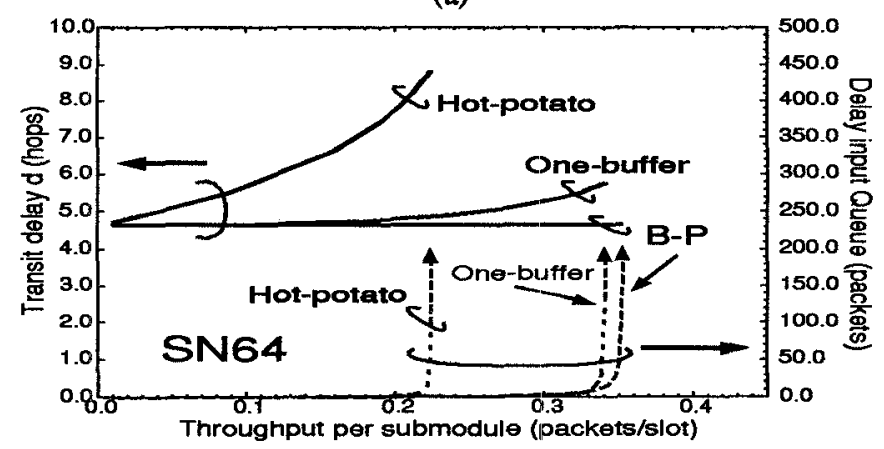

(b)

Figure 4: A cell's delay is the sum of its transit delay $d$, and its input queueing delay (in the input queue at the submodule where the cell originates). a) shows results for MS with 64 nodes. b) shows results for SN with 64 nodes.

where $\left\lceil\frac{m}{2}\right\rceil$ in (8) is the smallest integer larger than $\frac{m}{2}$ and SER is the sample error rate, that is the probability that a sample is in error. However, we will assume the worst-case scenario in which $R 1$ submodules communicate only with submodules of the same kind and the same holds for submodules of kind $R 2$. If submodule cross-communication is considered, the results with respect to transit delay, throughput and buffer-size tend to be similar to those presented in the previous sections. Therefore, we will assume that: 1) the head of the cells is transmitted at one common bit-rate $1 ; 2$ ) cells have the same spatial size; i.e. R2 cells will contain $m * M$ more bits than R1 cells; 3) each submodule transmit/receive/store at one bit rate only depending on its kind; 4) the submodules are uniformly distributed over the network. The total number of submodules per channel is $\aleph=N_{R 1}+N_{R 2}$. We will approximate the probability to find a cell in transit of bit-rate $\mathrm{R} 1\left(P_{p-R 1}\right)$ to the probability that one submodule chosen at random receives/transmits at R1 $\left(P_{n-R 1}\right)$, so that $P_{p-R 1}=P_{n-R 1}=\frac{N_{R 1}}{\aleph}$ and similarly for $R 2$ cells.

If there is a conflict in a submodule that receives at a bit-rate different from that of both cells, one of the cells is deflected, i.e. the probability that one $\mathrm{R} 1$ test cell is deflected at any submodule is $p_{R 1}=\frac{P_{c}}{(2)(2)} P_{p-R 1} P_{n-R 2}$, where $P_{c}=u(1-a)\left(1-P_{d c}\right)$ is the probability of having a care cell at the input of the routing switch (see Fig. 1b) together with a R1 test cell at an intermediate care submodule. The first $1 / 2$ refers to the probability of having two care cells with the same output preference, while the second $1 / 2$ is the probability that one of the two cells is selected for deflection. $P_{p-R 1}$ is the probability that the second care cell is R1 and $P_{n-R 2}$ is the probability that the submodule reads at $\mathrm{R} 2$. Note that the probability that one $\mathrm{R} 2$ test cell be deflected is $p_{R 2}=$ $\frac{P_{c}}{(2)(2)} P_{p-R 2} P_{n-R 1}$ which is numerically the same as $p_{R 1}$. 
When the buffered cells have access priority (B-P), the probability to store a cell in one buffer of an R1 submodule $\beta_{R 1}$ and the probability of transmitting a cell from one buffer of an R1 submodule $\mu_{R 1}$ is:

$$
\begin{aligned}
\beta_{R 1}= & \frac{\left(1-P_{d c}\right)^{2}}{(2)(2)}\left[A 2\left(2 P_{p-R 2} P_{p-R 1}+P_{p-R 1}^{2}\right)+g q_{R 1}^{2} A 1\right] \\
\mu_{R 1} & =\left(q_{R 1}+\frac{1-q_{R 1}}{2}\right)\left(A 0+A 1 P_{d c}\right)+\frac{A 1\left(1-P_{d c}\right)}{2} \\
& +\frac{1}{2} \frac{A 2\left(1-P_{d c}\right)^{2}}{2}
\end{aligned}
$$

An arrival $\left(\beta_{R 1}\right)$ will occur when there are two incoming care cells with the same output preference $(1 / 2)$ and with different bit rate or with same bit rate $\mathrm{R} 1$ as the submodule's bit rate $\mathrm{R} 1$, or the submodule generates a new care cell whenever the buffers are empty and there is one incoming care cell with the same output preference $(1 / 2)$. The second $1 / 2$ in (9) is the probability to store the cell in buffer 01 or $02 . \mu_{R 1}$ can be obtained reasoning as in (3) except that in this case $q_{R 1}=\frac{\mu_{R 1}-\beta_{R 1}}{\mu_{R 1}}$ is the probability that one of the buffers of a submodule that transmits/receives at $\mathrm{R} 1$ is empty. The probability to store a cell in one buffer of an $\mathrm{R} 2$ submodule $\left(\beta_{R 2}\right)$ and the probability of transmitting a cell from one buffer of an R2 submodule $\left(\mu_{R 2}\right)$ can be obtained by reasoning as in expressions (9) and (10) and in this case $q_{R 2}=\frac{\mu_{R 2}-\beta_{R 2}}{\mu_{R 2}}$.

The average number of newly transmitted cells per submodule is obtained as the probability of having a new cell times the probability that both buffers of an R1 submodule are empty $\left(q_{R 1}^{2} P_{n-R 1}\right)$ or both buffers of an $\mathbf{R} 2$ submodule are empty $\left(q_{R 2}^{2} P_{n-R 2}\right)$ times the probability that at least one of the two slots is free:

$$
\frac{\lambda}{\aleph}=g \chi\left(1-u^{2}(1-a)^{2}\right)
$$

where $\chi=q_{R 1}^{2} P_{n-R 1}+q_{R 2}^{2} P_{n-R 2}$. For this case the link utilization is

$$
u=\frac{\sqrt{a^{2}+(g \chi)^{2}(1-a)^{2}}-a}{(g \chi)(1-a)^{2}},
$$

and the total average normalized transit delay of an $\mathrm{R} 1$ test cell is

$$
d=H\left(1+\frac{D_{b-R 1} \rho_{R 1}}{W}\right)
$$

where $\rho_{R 1}=\frac{1}{2} u(1-a)\left(1-P_{d c}\right)^{2}\left(P_{p-R 2}+\frac{P_{p-R 1}}{2}\right)\left(P_{n-R 1}\right)$ is the probability of buffering an R1 test cell. Similarly the average delay of an R2 cell can be computed using (13) substituting the corresponding $D_{b-R 2}$ and $\rho_{R 2}=\frac{1}{2} u(1-a)\left(1-P_{d c}\right)^{2}\left(P_{p-R 1}+\right.$ $\left.\frac{P_{p-R 2}}{2}\right)\left(P_{n-R 2}\right)$. The queue size and queueing delay for $\mathrm{R} 1$ and R2 bit rate submodules are obtained using eqs. for $Q_{b}$ and $D_{b}$ substituting the respective $\mu$ and $\beta$ probabilities.

Fig. 5a shows the average queue size and average internal queueing delay in number of cells of a MS topology with 64 nodes. The average queue size is smaller than 1 cell and the average queueing delay is lower than 4 cells. Observe that theory and simulation results show a good agreement. Since SN topology(B-P) performs similarly, we only considered MS. Fig. $5 b$ shows throughput and

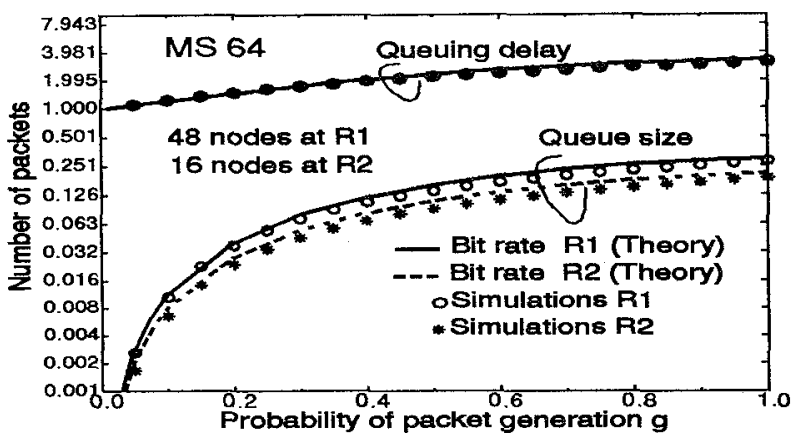

(a)

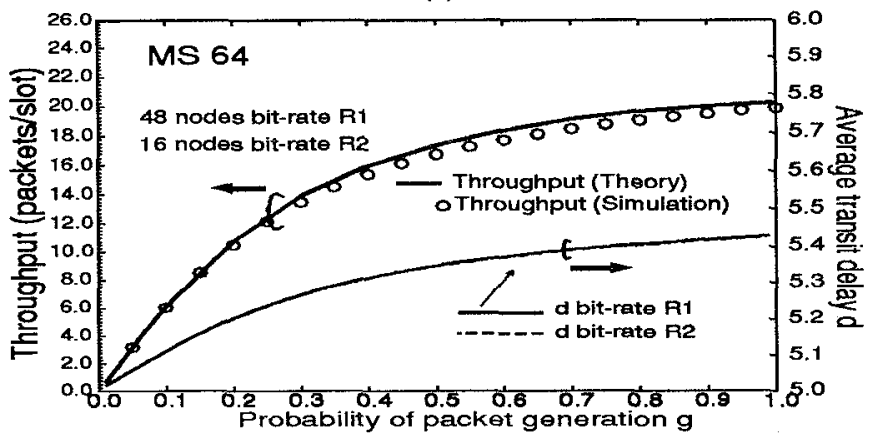

(b)

Figure 5: a) Queueing delay and Queue size in number of cells vs. $g$ for Manhattan Street and ShuffleNet topologies. b)Throughput and Total network cell delay in number of hops vs. $g$ for Manhattan Street with 48 nodes at bit rate R1 and 16 nodes at bit rate $R 2$.

average transit delay versus probability of cell generation. $\mathrm{Ob}-$ serve that due to deflections the throughput is slightly lower compared to the one in Fig. 2. Also, the average normalized delay $d$ increases $(0.4$ at $g=1)$ due to deflection of cells.

The simulations statistics of the two bit rate communication network were obtained computing the average of queue size, queuing delay and throughput from 10,000 different uniformly distributed random locations of the $R 1$ and $R 2$ submodules. The reason is that the teletraffic performance depends on the location of the R1 and $\mathrm{R} 2$ submodules. Also, each location of the $\mathrm{R} 1$ and $\mathrm{R} 2$ submodules was simulated for 30,000 clock cycles, after discarding 10,000 initial cycles to allow for transients to die out.

\section{DEVICE-INDUCED OPTICAL CROSSTALK}

The crosstalk generated in a $2 \times 2$ optical space switch is due to incomplete switching. A fraction $1-\alpha$ of the signal power exits from the desired port, while a fraction $\alpha$ leaks from the undesired port. If two signals at the same wavelength are present at the inputs, intra-band crosstalk is generated.

A wavelength DEMUX behaves like a prism, that fans out the light from the input fiber into distinct color (wavelength) beams, which are coupled to distinct outputs. The crosstalk in the DEMUX is (see Fig. 1a) due to residues of light from neighboring colors on each output. This inter-band crosstalk becomes intraband crosstalk at the multiplexer (MUX), when colors are merged again on the output fiber [13]. The inter-band crosstalk can be reduced by placing narrow-band optical filters before multiplexing. The amount of suppression of the inter-band components will depend on the transfer function $T(\Delta \lambda)$ of the filters. 


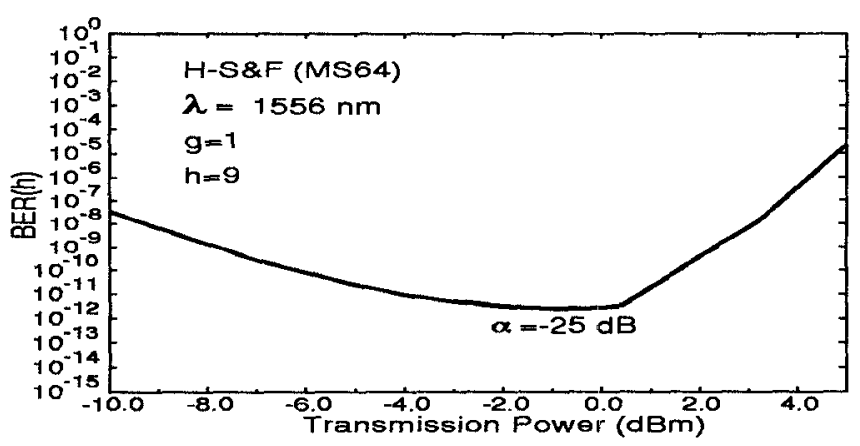

(a)

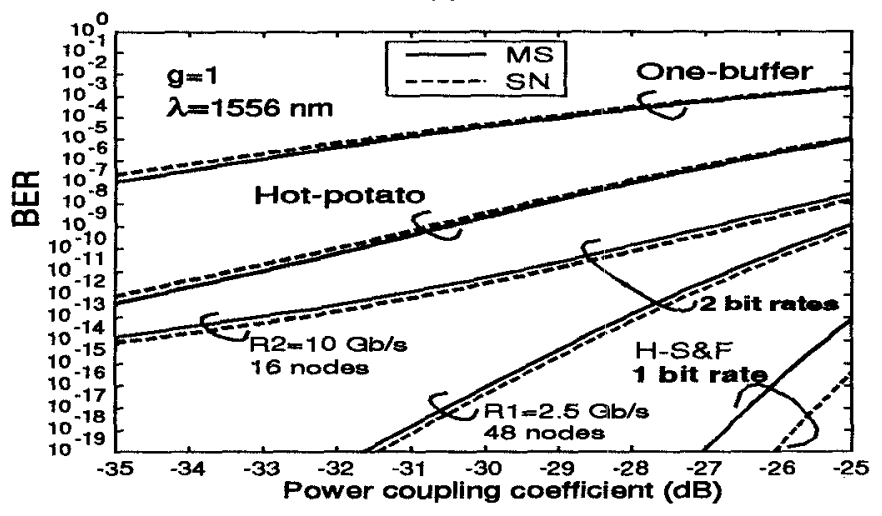

(b)

Figure 6: a) Conditioned BER(h) versus transmission power at hop number 9 , $\mathrm{g}=1$. b) BER versus power coupling coefficient $\alpha$ for $\mathrm{g}=1$.

\section{TRANSMISSION RESULTS}

In multi-hop networks the BER is obtained by conditioning the $B E R(h)$ on the number of hops $h$, where $h$ is a random variable, taken by a typical cell as $B E R=\sum_{h=1}^{\infty} B E R(h) P(h)$ [14]. The hop distribution $\mathbf{P}(\mathrm{h})$ [3] depends on network topology, routing, and load, while the conditional BER(h) depends on the traffic load and the optical characteristics of the network [5].

We analyzed a network with four channels in the range of 1550 $\mathrm{nm}$ to $1556 \mathrm{~nm}$, with $2 \mathrm{~nm}$ channel separation. DEMUX with adjacent signal inter-band crosstalk of $-30 \mathrm{~dB}$, and a $2 \times 2$ crossbar optical switch with coupling power coefficient $\alpha$ between $-25 \mathrm{~dB}$ and $-35 \mathrm{~dB}$ are assumed. Filters at the output of the main switch (see Fig. 1a) have a transfer function $T(\Delta \lambda)=-17 \mathrm{~dB}$. We represent each amplifier by using the spectrally resolved numerical model of [16] with a forward pumping scheme. The absorption and gain parameters are the same as those of fiber 2a in [17] with a length of $20 \mathrm{~m}$ and a pump power of $50 \mathrm{~mW}$. Thus, it is assumed that the EDFA's are operating in the saturated regime. A bandwidth of 125 $\mathrm{GHz}$ is used to resolve the effect of ASE spectrum. The optical filter at the receiver has a $0.2 \mathrm{~nm}$ bandwidth and the electrical filter has a $2.5 \mathrm{GHz}$ or $10 \mathrm{GHz}$ bandwidth depending on the kind of node $\left(N_{R 1}\right.$ or $\left.N_{R 2}\right)$. We assumed a fiber with dispersion coefficient $D_{c}=1 \mathrm{ps} / \mathrm{km}-\mathrm{nm}$, a loss coefficient of $0.2 \mathrm{~dB} / \mathrm{km}$, an internode distance of $15 \mathrm{~km}$, a total node loss of $12.5 \mathrm{~dB}$. The optical amplifiers are located at the output of each node.

We used a semi-analytical method [5], [15] to determine the error rate given that the path length $h$ is known. Fig. 6a shows the conditional BER(h) for hop number 9 versus transmission power at $\mathrm{g}=1$ for the channel at $1556 \mathrm{~nm}$, the one with the worst gain.

\begin{tabular}{|c|c|c|c|c|c|c|}
\hline & $\begin{array}{c}\mathrm{MS}, \mathrm{H}-\mathrm{S} \& \mathrm{~F} \\
\text { B-P }\end{array}$ & $\begin{array}{c}\text { MS, H-S\&F } \\
\text { N-P }\end{array}$ & $\begin{array}{c}\text { MS, H-S\&F } \\
\text { 2BR/B-P }\end{array}$ & $\begin{array}{c}\mathrm{SN}, \mathrm{H}-\mathrm{S} \& \mathrm{~F} \\
\text { B-P }\end{array}$ & $\begin{array}{c}\text { SN, H-S\&FF } \\
\text { N-P }\end{array}$ & $\begin{array}{c}\mathrm{SN}, \mathrm{H}-\mathrm{S} \& \mathrm{~F} \\
2 \mathrm{BR} / \mathrm{B}-\mathrm{P}\end{array}$ \\
\hline Iransit delay d at $\mathrm{g}=1$ & 5 & $\infty$ (sturation) & 5.4 & 4.7 & $\infty$ (saturation) & 5.1 \\
\hline Throughput & good & best & good & good & imbalance & good \\
\hline $\begin{array}{l}\text { Average } \\
\text { Quetre size }\end{array}$ & $<1$ slot & $\begin{array}{l}\text { saturation } \\
\text { at } g>0.95\end{array}$ & $<1$ slot & $<1$ slot & $\begin{array}{c}\text { imbalance } \\
\text { at } g>0.7\end{array}$ & $<1$ slot \\
\hline $\begin{array}{c}\text { Average } \\
\text { Queveing delad }\end{array}$ & $<4$ slots & $\begin{array}{l}\text { saturation } \\
\text { at } g>0.95\end{array}$ & $<4$ slots & $<4$ slots & $\begin{array}{l}\text { imbalance } \\
\text { at } g>0.7\end{array}$ & $<4$ slots \\
\hline BER, $\begin{array}{r}\alpha=-25 \mathrm{~dB} \\
\alpha=-27 \mathrm{~dB}\end{array}$ & $<10^{-9}$ & $<10^{-9}$ & 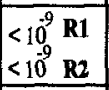 & $<10^{-9}$ & $-=-$ & 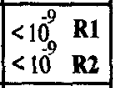 \\
\hline $\begin{array}{l}\text { Buffer } \\
\text { Imbalance }\end{array}$ & NO & PARTIAL, & NO & No & YES & NO \\
\hline
\end{tabular}

Table 1:Summary of results

Results are shown for MS topology with a coupling coefficient of $\alpha=-25 \mathrm{~dB}$ and a bit rate of $2.5 \mathrm{~Gb} / \mathrm{s}$. The main impairments considered to compute BER(h) are intra-band crosstalk and ASE noise. For low transmission power the predominant beat noise is signalASE that increases with bit rate and for high transmission powers the signal-crosstalk beat dominates, a noise that is bit rate independent.

Fig. 6b shows BER results against $\alpha$ for a network of 64 nodes at $g=1$. Figure shows results for one-buffer, hot-potato (from [5]) and H-S\&F. Observe that H-S\&F (1 bit rate) performs much better than one-buffer and hot-potatodeflection routing. The reason is that $\mathrm{H}-\mathrm{S} \& \mathrm{~F}$ has the minimal average propagation delay and therefore the impact of intraband crosstalk and ASEnoise is minimized. Note that BER of H-S\&F when all nodes transmit at $R=2.5 \mathrm{~Gb} / \mathrm{s}$ (one bit rate) is below $10^{-9}$ for any $\alpha$. Results for H-S\&F (one bit rate) are for N-P, however B-P performs very similar to N-P. Also Fig. $6 \mathrm{~b}$ shows results for the case of dual bit rate communication (B-P), when 48 nodes use bit rate $R 1=2.5 \mathrm{~Gb} / \mathrm{s}$ and 16 nodes use bit rate $R 2=10 \mathrm{~Gb} / \mathrm{s}$. In this case the BER of $2.5 \mathrm{~Gb} / \mathrm{s}$ nodes deteriorates because of possible cell deflections and the BER of 10 $\mathrm{Gb} / \mathrm{s}$ nodes is worse because of cell deflections and higher bit rate. However, the BER is reasonably low for values of $\alpha$ below -27 dB. The curves for H-S\&F were computed neglecting electronic regeneration of buffered cells, and are thus upper bounds on the actual $B E R$ values.

\section{CONCLUSIONS}

We presented a node architecture with electronic routing buffers. Cells are stored just in the case of conflict to avoid deflection, otherwise they traverse the node without opto-electronic conversion (transparent cut-through [6] routing). Table 1 summarizes the results of this analysis. It is shown that cell correlation is much more severe in SN64 than in MS64. When locally generated cells have access priority, internal buffers can reach saturation at high loads $(g>0.95)$ for MS and $(g>0.7)$ for SN topologies. Our results show that H-S\&F performs better than hot-potato and onebuffer deflection routing in terms of throughput, propagation delay, and BER. In terms of throughput, N-P H-S\&F performs better than B-P H-S\&F and deflection routing. Also, B-P H-S\&F performs slightly better than one-buffer deflection routing and better than hot-potato deflection routing. In terms of propagation delay, one of the main advantages of H-S\&F is that it provides the minimum average propagation delay. Also, the results show that under 
SN and MS topologies with 64 nodes the BER is always lower than $10^{-9}$ assuming no regeneration of a test cell, $\alpha=-25 \mathrm{~dB}$, and one bit rate communication scheme. Moreover, it is shown that with a combination of deflection routing and hybrid store-and-forward the network can accommodate communication with two different bit rates. When 48 nodes use bit rate $R 1=2.5 \mathrm{~Gb} / \mathrm{s}$ and 16 nodes use bit rate $R 2=10 \mathrm{~Gb} / \mathrm{s}$, the BER of $2.5 \mathrm{~Gb} / \mathrm{s}$ nodes is lower than $10^{-9}$ for $\alpha=-25$ and the BER of $10 \mathrm{~Gb} / \mathrm{s}$ nodes is lower than $10^{-9}$ for $\alpha$ below $-27 \mathrm{~dB}$.

\section{APPENDIX A}

In this section we briefly explain how the analysis extends to analyze the network with input queues. In the model we describe in Section 2 and subsequently analyzed, If a new cell is generated but can not be injected into the network, local blocking occurs and the local cell is discarded. An alternative procedure would be to queue the new cell until they can be admitted into the network. Such input queueing results in a possible queueing delay for packets as they reach the submodule.

Assume that the number of new packets that arrive at a submodule during a time slot has a Poisson distribution with parameter $\lambda^{\prime}$ where $\lambda^{\prime}>0$, and that the number of arrivals from node to node and slot to slot are independent. If the system is stable then the steady-state throughput per node per channel will also be $\lambda^{\prime}$ [18]. Then the throughput per node per channel given by equation 4 is equal to $\lambda^{\prime}$. Now as an example we consider the case in which internal buffers have access priority over the new cells generated by the submodule, then the number of new cells that can be potentially injected at a submodule in a time slot has the binomial distribution $\operatorname{Bin}\left(1, q^{2}\left(1-u^{2}(1-a)^{2}\right)\right)$. Now by the independence assumption that the number of packets that can be potentially injected from slot to slot are independent, the queue of packets waiting at a submodule can be model as a discrete time queue [18] where the number of arrivals in each slot is Poisson with mean $\lambda^{\prime}$ and the number of potential services in each slot has the binomial distribution $\operatorname{Bin}\left(1, q^{2}\left(1-u^{2}(1-a)^{2}\right)\right)$.

Now using equation (4.1) of [18] as an approximation to the waiting time in an input queue with Poisson arrivals, input queue delay is $D_{I-B}=\frac{\lambda^{\prime}+2 m}{2\left(1-m-\lambda^{\prime}\right)}$ where $\lambda^{\prime}=g q^{2}\left(1-u^{2}(1-a)^{2}\right)$, $m=1-q^{2}\left(1-u^{2}(1-a)^{2}\right)$ for the case the internal buffers have access priority (B-P) over the new cells and $\lambda^{\prime}=g\left(1-u^{2}(1-a)^{2}\right)$, and $m=u^{2}(1-a)^{2}$ when access priority is given to locally generated cells (N-P).

\section{References}

[1] N. F. Maxemchuk, "Comparison of deflection and store-andforward techniques in the manhattan street and shuffie-exchange networks," in Proc. IEEE INFOCOM '89, pp. 800-809, Apr. 1989.

[2] R. Krishnan and N. F. Maxemchuk, "Is there life beyond linear topologies? a comparison of DQDB and the manhattan street network," in Proc. IEEE INFOCOM'93, pp. 690-698, 1993.

[3] F. Forghieri, A. Bononi, and P. Prucnal, "Analysis and comparison of hot-potato and single-buffer deflection routing in very high bit rate optical mesh networks," IEEE Trans. on Commun., vol. 43. no. 1, pp. 88-98, January 1995.

[4] E. L. Goldstein, L. Eskildsen, and A. Elrefaie, "Performance implications of component crosstalk in transparent lightwave networks," IEEE Photon. Technol. Lett., vol. 6, no. 5, pp. 657-660, 1994.
[5] G. A. Castañón, O. K. Tonguz, and A. Bononi, "BER performance of multi-wavelength optical cross-connected networks with deflection routing," IEE Proc.-Communications, vol. 144, no. 2, pp. 114120, April 1997.

[6] P. Kermani and L. Kleinrock, "Virtual cut-through: a new computer communication switching technique," Computer Networks, vol. 3, no. 4, pp. 267-286, 1979.

[7] N. F. Maxemchuk, "Regular mesh topologies in local and metropolitan area networks," AT\&T Tech. J., vol. 64, no. 7, pp. 1659-1685, Sept. 1985.

[8] M. Eisenberg and N. Mehravari, "Performance of the multichannel multihop lightwave network under nonuniform traffic," IEEE J. Select. Areas Commun., vol. 6, no. 7, pp. 1063-1078, August 1988.

[9] A. K. Choudhury and V. O. K. Li, "An approximate analysis of the performance of deflection routing in regular networks," IEEE J. Select. Areas Commun., vol. 11, no. 8, pp. 1302-1316, October 1993.

[10] A. G. Greenberg and J. B. Goodman, "Sharp approximate models of deflection routing in mesh networks," IEEE Trans. Commun., vol. 41, no. 1, pp. 210-223, Jan. 1993.

[11] L. Kleintock, Queueing Systems, Vol. 1: Theory. New York: Wiley, 1975.

[12] A. S. Acampora and A. Shah, "Multhop lightwave networks: a comparison of store-and-forward and hot-potato routing," IEEE Trans. on Commun., vol. 40, pp. 1082-1090, June 1992.

[13] J. Zhou, R. Cadeddu, E. Casaccia, C. Cavazzoni, and M. J. O'Mahony, "Crosstalk in multi-wavelength optical cross-connect networks," IEEE J. Lightwave Technol., vol. 14, no. 6, pp. 14231435, 1996.

[14] A. Bononi, F. Forghieri, and P. Prucnal, "Design and channel constrain analysis of ultrafast multihop all-optical networks with deflection routing employing solitons," IEEE J. Lightwave Technol., vol. 11, no. 12, pp. 2166-2176, Dec. 1993.

[15] G. A. Castañón, O. K. Tonguz, and A. Bononi, "Transmission performance of transparent multi-wavelength optical cross-connected networks," in IEEE Symposium on Computers and Communications (ISCC'97), vol. 1, pp. 323-329, 1-3 July, 1997. Also available at http://www.acsu.buffalo.edu/castanon/publications.html.

[16] C. R. Giles and E. Desuvire, "Modeling erbium-doped fiber amplifiers," IEEE J. Lightwave Technol., vol. 9, no. 2, pp. 271-283, Feb. 1991.

[17] C. R. Giles, C. Burrus, D. DiGiovanni, N. Dutta, and G. Raybon, "Characterization of erbium-doped fiber and application to modeling 980-nm and 1480-nm pumped amplifiers," IEEE Photon. Technol. Lett., vol. 3, pp. 363-365, 1991.

[18] A. G. Greenberg and B. Hajek, "Deflection routing in hypercube networks," IEEE Trans. Commun., vol. 40, no. 6, pp. 1070-1081, June 1992. 